\title{
The Influence of DMBA (7, I 2-dimethylbenz-[a]anthracene) Regimen In The Development of Mammae Carcinogénesis on Sprague Dawley Female Rat
}

\author{
Agung Eru Wibowo ${ }^{1, *}$, Sriningsih $^{1}$, Puspita Eka Wuyung ${ }^{2}$, Raafqi Ranasasmita ${ }^{3}$ \\ ${ }^{1}$ Center for Pharmaceutical and Medical Technology - BPPT \\ ${ }^{2}$ Department of Pathology and Anatomy- Faculty of Medicine, Indonesia University \\ ${ }^{3}$ Department of Biochemistry-Bogor Agricultural University ${ }^{3)}$.
}

\begin{abstract}
There are many methods for development of mammae cancer animal model, one of which is chemical induction using carcinogenic agent, DMBA. This research aimed to explore the influence of dose and time regimens of DMBA on development of mammae carcinogenesis on Sprague dawley female rats. The first study was 50 rats treated with $20 \mathrm{mg} / \mathrm{kg}$ bw of DMBA orally for eleven times at twice a week. Morphological evaluations were conducted with mammae palpation for 15 weeks and then all of rats were sacrificed for collecting mammae organs for histological analysis using hematoxylin-eosin staining. The results showed that the first and the latest nodules appeared at the fourth-week and the fourteenth-week after ending DMBA induction, respectively, in which the most often nodule appearances were at the seventh-week. The number of nodule incidence and multiplicity were by $74 \%$ and 2 noduls/rat, respectively. Histological analysis of mammae glands determined that they fell under in Ductal Carcinoma Invasive (DCIV) category. The second study was 25 rats gavaged orally with DMBA at dose $20 \mathrm{mg} / \mathrm{kg}$ bw for five times every three days. After palpating for 15 weeks, the results showed that no nodule was observed but the histological analysis demonstrated developing of mammae gland carcinogenesis reaching about 60\% Ductal Carcinoma Insitu (DCIS) and 40\% Ductal Carcinoma Invasive (DCIV) stages. Based on the results of this study can be concluded that the dose and frequency of DMBA will affect the successful development of mammary gland carcinogenesis. In DMBA induction with low frequency, no data showed the incidence and multiplicity of tumor, but histopathologic level carcinogenesis can be distinguished. In DMBA induction with high frequency, incidence and multiplicity of tumor data can be obtained but can not be distinguished histopathologically.
\end{abstract}

Keywords: DMBA, doses, carcinogenesis, mammae, histopatology

\section{INTRODUCTION}

WHO in the world health report 2008 estimates that in 2030 the cancer disease is one cause of death world community that the tendency to increase. WHO and the World Cancer Prevention Organisation (Union Internationale Contre le Cancer, UICC), also approximate that will increase the incidence of cancer in the world as much as $300 \%$ in 2030 , where $70 \%$ of them occur in developing countries. This happens in line with the changing lifestyle of the people (WHO, 2009). In Indonesia, the prevalence of cancers also tends to increase. Based on the medical record data of Dharmais Cancer Hospital (RSKD), breast and cervical cancer are the two biggest cases handled by RSKD (RSKD, 2010). The high breast cancer cases encourages the need for research to find a solution on early detection, prevention, treatment and management of the right to suppress the emergence of cases, treat and keep the quality of life for sufferers.

Chemoprevention is the use of substances (agents) chemistry of natural, synthetic or biological to restore, suppress, or prevent carcinogenic progression that can become invasive cancer. Chemoprevention is one rational and attractive strategy for development (Tsao et al, 2004).

*Corresponding author e-mail : bowoae@yahoo.com 
Chemoprevention based on the concept of the nature of multifocal (many aspects) and multistep (many steps) in the process of carcinogenesis. The concept of carcinogenesis was used as an approach in the development of animal models for chemoprevention research or the development of anticancer compounds. Associated with breast cancer, one model that has been widely used is the induction of carcinogenic substances in rodent species. Carcinogenic substance used for the induction of mammary gland carcinogenesis in animals was 7.12-dimethylbenz-[a] anthracene (DMBA) (Fig. 1). DMBA is a compound class of polyaromatic hydrocarbons ( $\mathrm{PAH})$, which is a carcinogen and has similar properties to estrogen. DMBA compound is procarcinogen which will become active metabolite after metabolized by the cytochrome P-450 and bind covalently with DNA to form DNA adducts which in turn can trigger the formation of malignant (Lenoir, et al, 2005).<smiles>Cc1c2ccccc2c(C)c2c1ccc1ccccc12</smiles>

Figure I. The structure of 7.I2-Dimethylbenz [a] anthracene (DMBA) (Sigma, 2007)

Interaction between DNA with carcinogenic hydrocarbon compounds which are catalyzed by the enzyme system (Fig. 2), ie enzymes microsomes and enzyme-hydroxylation in liver which will convert the compound benzo [a] pyrene into active intermediates that can bind covalently with DNA (Zeiger R.S, 1972).

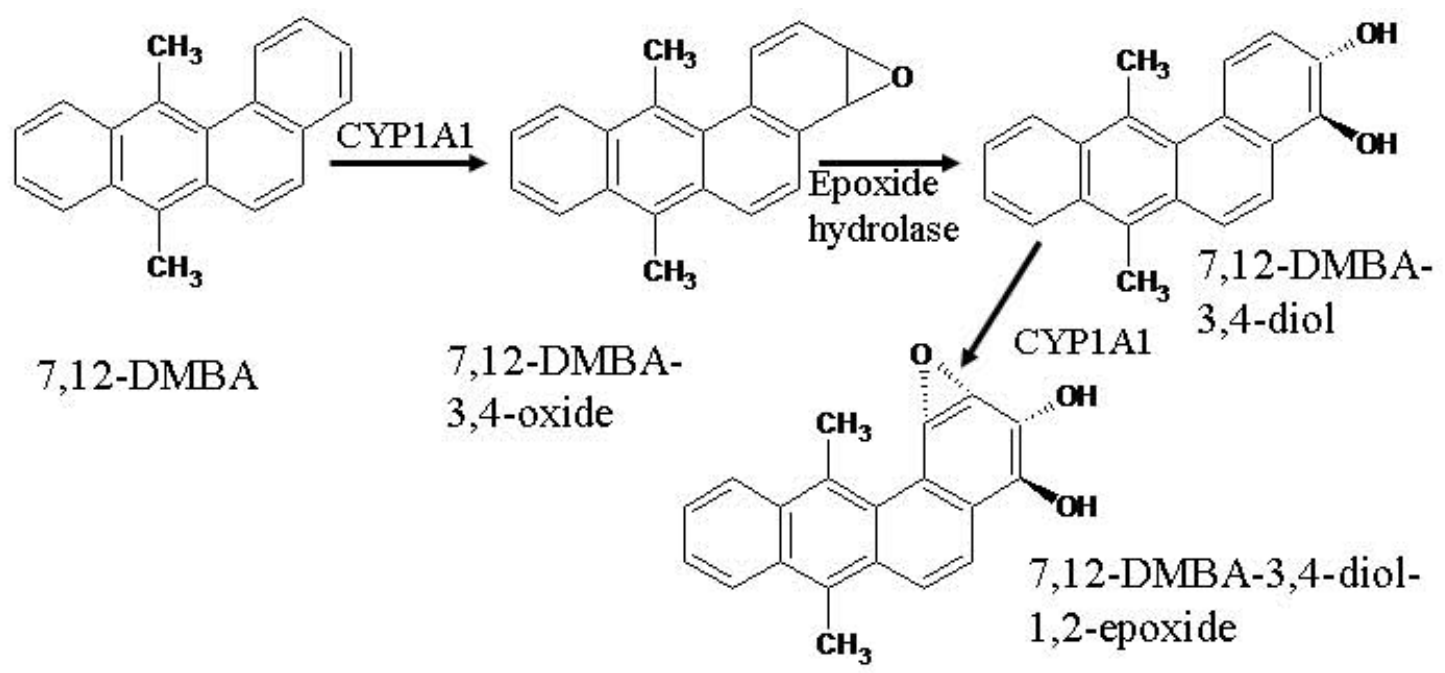

Figure 2. Metabolic activation of DMBA to carcinogenic metabolites of 7.12-DMBA-3,4-diol and to 7.12DMBA-3, 4-diol-I ,2-epoxide by the enzyme CYPIAI and epoxide hydrolase (Androutsopoulos et al, 2009).

Induction of CYP1A1 expression is mediated through specific receptors cytosolic, namely aryl hydrocarbon receptor (AHR). These receptors are part of the protein that can bind cytosolic of environmental contaminants such as polycyclic aromatic hydrocarbon compounds
(PAHs) and derivatives halogen compounds (Androutsopoulos et al, 2009). In the development of research on animal carcinogenesis should be noted that there are several factors that can affect the success of the induction of mammary gland carcinogenesis, including dosage and frequency of 
induction of carcinogen. This research was conducted to provide information about the effect of dosage and frequency of induction of DMBA in Sprague Dawley female rat against incidence and multiplicity of tumor and histopathologicaly carcinogenesis.

\section{MATERIAL AND METHOD}

\section{Materials}

Female rats strains Sprague Dawley (SD) age 27 days, obtained from the Food and Drug Research Center - BPOM (F1 generation from the parent who brought from Japan Shizouka Laboratory Center, SLC). 7,12-dimetilbenz [a] anthracene, DMBA (Sigma); Carboxyl Methyl Cellulose-Na, Na CMC (technical); feed obtained from PD. Kasman Jakarta; Sodium thiosulfate (Merck), $\mathrm{K}_{2} \mathrm{HPO}_{4}$ (Merck), $\mathrm{KH}_{2} \mathrm{PO}_{4}$ (Merck); Hematoxylin, eosin; Xylol (Merck); Ethanol pa (Merck); formalin; $\mathrm{Na}_{2} \mathrm{HPO}_{4}$ (Merck), $\mathrm{NaH}_{2} \mathrm{PO}_{4}$ (Merck).

\section{Method:}

Group I. Prepared 50 strains of female rats Sprague Dawley (SD) age 4 weeks, and separated into 5 groups of 10 rats each, and then adapted to the conditions of experimental cages for 2 weeks. Rats were given food and drink ad libitum. DMBA dissolved in corn oil $(2 \mathrm{mg} / \mathrm{mL})$ with the aid of vortex. Induction is done intra gastric at a dose of $20 \mathrm{mg} / \mathrm{kg}$, carried 11 times (twice a week). After the induction is complete, tumor growth were observed in rat mammary region by palpation.

Group II. Prepared 25 strains of female rats Sprague Dawley (SD) age 4 weeks, and separated into 5 groups of 5 rats each, and then adapted to the conditions of experimental cages for 2 weeks. DMBA dissolved in corn oil $(2 \mathrm{mg} / \mathrm{mL})$ with the aid of vortex. Induction done intra gastric at a dose of $20 \mathrm{mg} / \mathrm{kg}$, performed 5 times (every 3 days). After the induction was complete, tumor growth were observed in rat mammary region by palpation.

\section{Histopathological Observations}

Making preparations for histology and stained with Hematoxylin and Eosin preparations carried out in accordance with the protocol that prepared by the Department of Pathology and Anatomic Faculty of Medicine, University of Indonesia. To analyze the level of carcinogenesis by scoring the level of carcinogenesis that occurs in tumor nodules. There are five scores carcinogenesis level: score 0 (zero) for normal, score 1 for mild hyperplasia, score 2 for severe hyperplasia, score 3 for hyperplasia with athypia, score 4 for Ductal Carcinoma In situ (DCIS) and score 5 for Ductal Carcinoma Invasive (DCIV) (Ting et al., 2007).

\section{RESULT}

The development of rat mammary gland carcinogenesis in group I (DMBA induction at doses of $20 \mathrm{mg} / \mathbf{k g}$, I I times).

In group $\mathrm{I}$, the observation of appearance and number of rat noduls was done for 15 weeks after the last induction. A total of 50 female rats were induced with DMBA, 37 of which appear nodules. The number of total nodules that emerged during 15 weeks of observation was 74 nodules (Fig. 3). Nodules first appeared at week 4 after the last DMBA induction (2 rats). Last nodules appeared at week 14 after the last DMBA induction (4 rats). Number of rats appears most visible nodules at week 7 after the last DMBA induction (8 rats). From the results it can be calculated values of tumor incidence was $74 \%$ and tumor multiplicity was 2 noduls/rat (Fig. 4). Complete data of the incidence and multiplicity of tumors in each group can be seen in Table 1 and Fig. 5. Histopathologic analysis of tumor nodules with HE staining showed that as many as $4 \%$ of treatment group had 4 score or Ductal Carcinoma In situ (DCIS) category and $96 \%$ of it achieved a 5 score or Ductal Carcinoma Invasive (DCIV) category. 


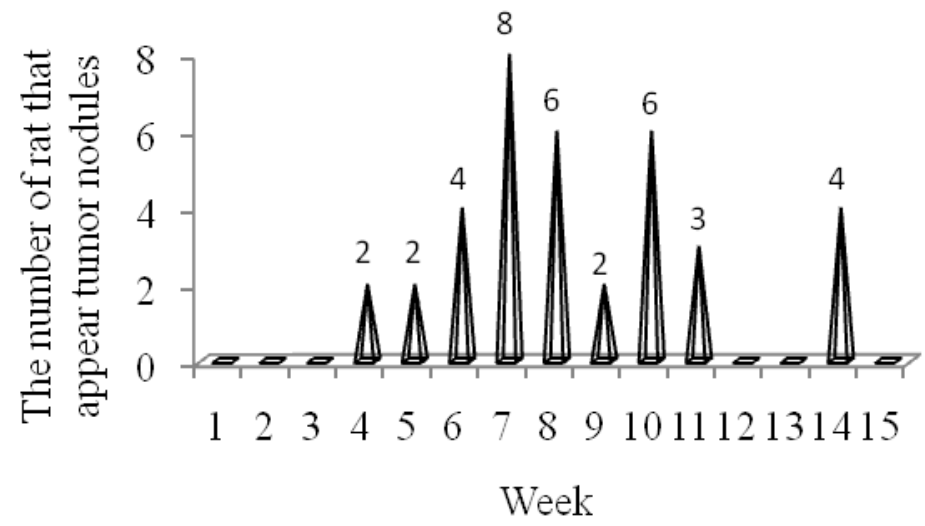

Figure 3. The number of rat that appear tumor nodules

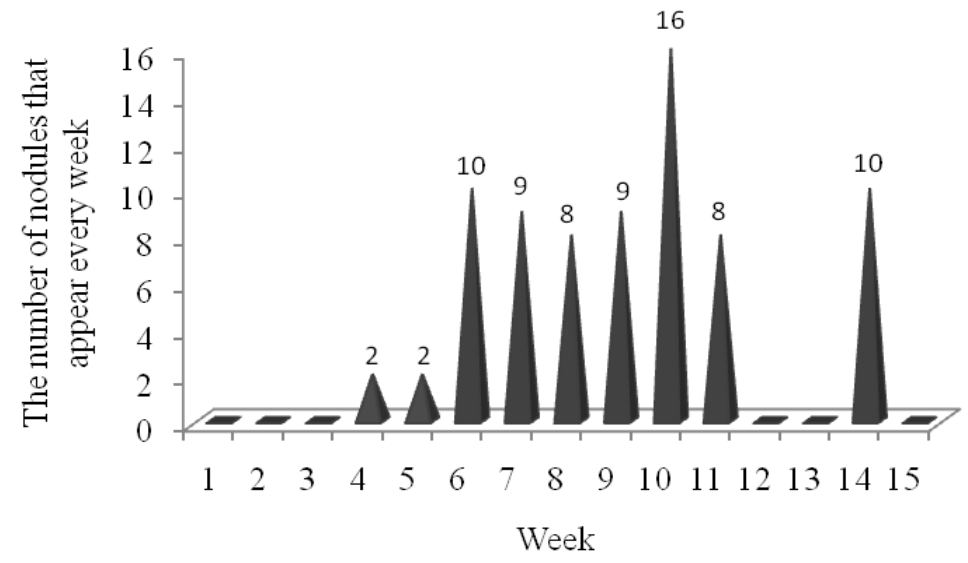

Figure 4. The number of nodules that appear every week

The development of rat mammary gland carcinogenesis in group II (DMBA induction

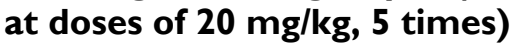

In the study group II, as many as 25 strains of SD female rats were induced with DMBA at a dose of $20 \mathrm{mg} / \mathrm{kg}$, given orally once 3 days 5 times. Observations nodules that emerged was done for 15 weeks after the last DMBA induced by palpation. Results showed up to the end of the observation no nodules were observed by palpation. Subsequently all the animals try to do surgery to take the mammary gland and histopathological analysis with $\mathrm{HE}$ staining. The results show that there have been processes of mammary gland carcinogenesis in rats, that is score 4 (Ductal Carcinoma in situ, DCIS) of $60 \%$ and score 5 (Ductal Carcinoma Invasive, DCIV) as much as $40 \%$. 

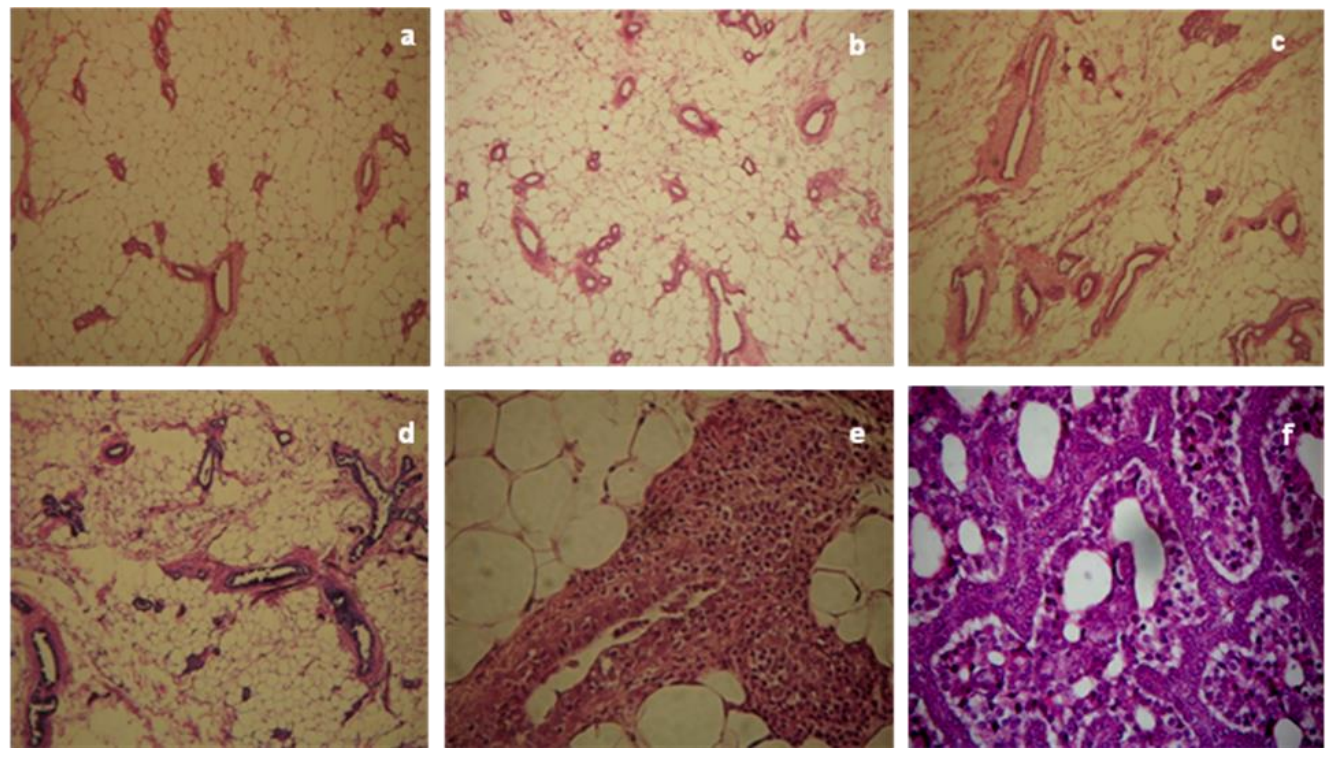

Figure 5. Score of carcinogenesis development: $0=$ normal (a), $I=$ mild hyperplasia $(b), 2=$ severe hyperplasia (c), 3 = hyperplasia with athypia (d), 4 = Ductal Carcinoma In situ (DCIS) (e), 5 = Ductal Carcinoma Invasive (DCIV) (f)

Table I. Value of tumor incidence and multiplicity of SD strain female rats by DMBA at dose of $20 \mathrm{mg} / \mathrm{kg}$ in II times frequency

\begin{tabular}{cccc}
\hline Group & Number of rat & $\begin{array}{c}\text { Incidence } \\
\text { (\%) }\end{array}$ & $\begin{array}{c}\text { Multiplicity } \\
\text { (nodul/rat) }\end{array}$ \\
\hline I & 10 & 70 & 1,86 \\
II & 10 & 40 & 2,50 \\
III & 10 & 70 & 2,29 \\
IV & 10 & 90 & 2,11 \\
V & 10 & 100 & 1,60 \\
Mean & 10 & $\mathbf{7 4}$ & $\mathbf{2 , 0 7}$ \\
\hline
\end{tabular}

\section{DISCUSSION}

One method of testing in vivo to see the effect of a sample on the growth of breast cancer is to use an animal model induced with carcinogenic materials. Carcinogens are compounds or materials that can cause cancer. Carcinogenesis is the process of developing cancer caused by carcinogenic substances. Carcinogenesis is a multistep process, which occurs in several stages of initiation, promotion and progression. Many factors can affect the process of carcinogenesis, including the dosage and frequency of administration of carcinogenic material. This research was conducted to provide information about the effect of dosage and frequency of induction of DMBA in Sprague Dawley female rat againts incidence and multiplicity of tumor and histopathologically carcinogenesis description.
In this study, experimental animals used were female rats of Sprague Dawley (SD) strain and carcinogenic materials used were 7.12dimethylbenz [a] anthracene (DMBA), a carcinogenic material from the class of compounds polyaromatic hydrocarbons (PAH), which has been widely used as an inducer of mammary cancer growth in mice. The consideration of SD strain female rats was used as test animals were (i). These animals are commonly used as test animals and has many scientific publications about carcinogenesis studies using strains of SD rats, (ii). Various publications reported success rate of induction of carcinogen in animal species is quite good and (iii). Easy in the provision and maintenance. In this study, female rats aged 6 weeks (42 days) during induction. Mammary gland susceptibility to carcinogenic substances depends on the age of the animal, around the age of 40-60 days after birth. At the age of the rats entering 
puberty earlier with the level of proliferation of terminal end buds (TEBs) high in mammary gland (Kubatka et al., 2002).

DMBA was chosen as the carcinogen material with consideration are: (i). This compound is rapidly metabolized into reactive carcinogens to form DNA adducts, (ii). Various studies have shown a high success rate in developing carcinogenesis using these compounds and (iii), receptors of this compound is the arylhydrocarbon (AhR) are largely contained in the reproductive glands, particularly the mammary gland. AhR is a transcription factor activated by a ligand-responsive compounds from nature. AhR many expressed in the reproductive tract in various species and several indications show that the AhR plays an important role in reproductive physiology (Pocar et al., 2005).

There are many endogenous factors of experimental animals affecting the carcinogenesis development level. Metabolism, hormonal and various immune system will affect the process of mammary gland carcinogenesis. These factors will affect the process of DMBA metabolism to be active metabolites and the next stage of carcinogenesis (initiation, promotion until progression). Interactions between metabolites of carcinogens with DNA to form DNA adducts also take time and involve various enzymes (mycromsomal and hydroxylation in liver), so that the process of carcinogenesis in each individual animal is not the same.

The study of mammary gland carcinogenesis in rat has noticed a great time to get maximum effect from an exposure to carcinogens. Window of susceptibility is a major factor in cancer risk. Window of susceptibility defined as the time in early puberty in which the mammary gland morphology is very sensitive to an initiation. In the carcinogenesis of mammary gland, mammary gland development stage showed different sensitivity to different initiation (Gear, 2007).

Food diet (high fat content) can affect the process of carcinogenesis. Hakkak et al (2005) reported that obesity increases the vulnerability of female mice strains Zaker against DMBA induction. This statement supports the hypothesis that obesity plays an important role in carcinogenesis (Hakkak et al., 2005).

From the results of the study in both groups showed that a dose of induction similar but different frequencies will provide different levels of carcinogenesis. Referring to the two parameters namely morphological observation (incidence and tumor mulitplicity) and histopathological parameters of the two groups can be obtained some results as follows:

Group I given with DMBA of $20 \mathrm{mg} / \mathrm{kg}$ bw (11 times frequency, 2 times a week) appeared $74 \%$ of tumor incidence. This meant that $74 \%$ of all experimental animals appeared tumor nodules. These results are lower than that of earlier researcher. Meiyanto et al., (2007) reported that induction of DMBA at a dose of $20 \mathrm{mg} / \mathrm{kg}$ as many as 10 times orally (2 times a week) and the $100 \%$ tumor incidence emerged at the $14^{\text {th }}$ weeks after the last induction. Research was conducted by Kubatka et al. (2002) provided that there was influence time of conducting research and the tumor incidence. Rats that treated with DMBA dose of $10 \mathrm{mg} / \mathrm{kg}$ orally as many as three times would produce $95.8 \%, 100 \%$ and $81.3 \%$ in the period of April to July, December to June and July to December, respectively (Kubatka et al., 2002).

Based on histopathological analysis by using Hematoxylin-Eosin (HE) staining, almost all of groups appeared neoplastic nodules in which almost $96 \%$ of rats was in the most severe levels of carcinogenesis by a score of 5, Invasive Ductal Carcinoma (DCIV) category and as much as $4 \%$ of them had the score of 4, Ductal Carcinoma Insitu (DCIS) category (Table 1). From these results we conclude that the HE-stained histopathology can unable to be used as differentiate levels of carcinogenesis because almost all nodules showed the same histopathology category.

In group II, until the end of the observation time there was not detected the presence mammary nodules, so that the DMBA inductive method at a dose of $20 \mathrm{mg} / \mathrm{kg}$ orally each three days in five times frequency could not be obtained morphological parameters (incidence and multiplicity of tumors). However, histopathologically by HE staining animals produced neoplastic changes that almost $60 \%$ of rats was in the score of 4, Ductal Carcinoma Insitu (DCIS) category and $40 \%$ of them was at scores of 5, Ductal Carcinoma Invasive (DCIV) category. Thus the induction of group II we can deduced that histopathological parameters can be used to analyze differences in levels of carcinogenesis without morphology description.

\section{CONCLUSION}

From these study we can driven a conclution that DMBA induction frequency determined the success of the mammary gland carcinogenesis process in Sprague dawley female 
rats. Giving DMBA at dose of $20 \mathrm{mg} / \mathrm{kg}$ bw in 11 times frequency will be obtained morphological parameter description such as incidence and multiplicity of tumors but the category of almost all nodules fell at the level of Ductal Carcinoma Invasive (DCIV) so that they was not be able to be distinguished each other. Otherwise at dose of 20 $\mathrm{mg} / \mathrm{kg}$ bw in 5 times frequency was gained histopathologically carcinogenesis level without morphological data like incidence and multiplicity of tumors.

\section{ACKNOWLEDGMENTS}

1. Center for Pharmaceutical and Medical Technology-BPPT which has provided space and facilities as well as support materials for research

2. Department of Pathology and Anatomic, Faculty of Medicine, University of Indonesia, which has helped in the histopathologically preparation and analysis

3. Okpri Meila and Dilla Meyfiana Erniawan for technical support during the experiments

\section{REFERENCES}

Androutsopoulos, V.P., Tsatsakis, A.M. and Spandidos, D.A., 2009, Cytochrome P450 CYPIAI: wider roles in cancer progression and prevention, BMC Cancer 9, 187.

Gear, R.B., Yan, M., Schneider, J., Succop, P., Heffelfinger, S.C., Clegg, D.J., 2007, Charles River Sprague Dawley Rats Lack Early Age-Dependent Susceptibility to DMBA-Induced Mammary Carcinogenesis, Int J Biol Sciences, 3(7), 408-4I6.

Hakkak, R., Holley, A.W., MacLeod, S.L., Simpson, P.M., Fuchs, G.J., Jo, C.H., KieberEmmons, T. and Korourian, S., 2005, Obesity promotes 7,12dimethylbenz(a)anthracene-induced mammary tumor development in female Zucker rats, Breast Cancer Research, 7, R627-R633.

Kubatka, P., Ahlersova, E., Ahlers, I., Bojkova, B., Kalicka, K., Adamekova, E., et al., 2002,
Variability of Mammary Carcinogenesis Induction in Female Sprague-Dawley and Wistar: Han Rats: the Effect of Season and Age, Physiol Res., 5, 633-640.

Lenoir, V., Canonico, M.B.Y., Perrin, M.H., Martin, A., Scholler, R., et al., 2005, Preventive and curative effect of melatonin on mammary carcinogenesis induced by dimethylbenz[a]anthracene in the female Sprague-Dawley rat. Breast Cancer Res., 7, R470-R476.

Meiyanto, E., Tasminatun, S., Susilowati, S., Murwanti, R. and Sugiyanto, 2007, Efek kemopreventif ekstrak etanolik Gynura procumbens (Lour), Merr pada karsinogenesis kanker payudara tikus. Majalah Farmasi Indonesia, I 8(3).

Pocar, P., Fischer, B., Klonisch, T. and Klonisch, S.H.P., 2005, Molecular interactions of the aryl hydrocarbon receptor and its biological and toxicological relevance for reproduction, Review, Society for Reproduction and Fertility, 28, I74I-7899.

RSKD, Laporan Bagian Rekam Medis RSKD. http://www.dharmais.co.id /index.php Istatistic-center.html. accesed on March 31,2010

Sigma-Aldrich, 2007. 7,I2-Dimethylbenz[ $\alpha]$ anthracene.http://www.sigmaaldrich.co m, accessed on April 27, 2007.

Ting, A.Y., Kimler, B.F., Fabian, C.J. and Petroff, B.K., 2007, Characterization of a preclinical model of simultaneous breast and ovarian cancer progression, Carcinogenesis, 28(I), I30-135,

Tsao, A.S., Kim, E.S. and Hong, W.K., 2004, Chemoprevention of Cancer, CA Cancer J Clin; 54, I50-I80.

World Health Organization. The World Health Report 2008: primary health care now more than ever. www.who.int/entity/whr/2008/08, accesed on August 9, 2009.

Zeiger, R.S., Salomon, R., Kinoshita, N. and Peacock, A.C., 1972, The Binding of 9,10Dimethyl-I,2-benzanthracene to Mouse Epidermal Satellite DNA in Vivo, Cancer Research, 32, 643-647. 